\title{
Upregulation of miR-184 Enhances the Malignant Biological Behavior of Human Glioma Cell Line A172 by Targeting FIH-1
}

\author{
Qinghua Yuan ${ }^{\mathrm{a}}$ Weida Gao ${ }^{\mathrm{a}}$ Bo Liu ${ }^{\mathrm{b}}$ Wei Yea \\ aDepartment of Neurosurgery, The Second Affiliated Hospital of Harbin Medical University, Harbin, \\ bDepartment of Neurosurgery, Daqing Oilfield General Hospital, Daqing, PR China
}

\section{Key Words}

$\mathrm{FIH}-1 \cdot$ Human glioma cancer • miR-184 • Malignant biological behavior

\begin{abstract}
Background: In recent years, miRNAs have been suggested to play key roles in the formation and development of human glioma. The aim of this study is to investigate the effect and mechanism of miR-184 expression on the malignant behavior of human glioma cells. Methods: The relative quantity of miR-184 was determined in human glioma cell lines, and the expression of hypoxia-inducible factor- 1 alpha (HIF-1 $\alpha$ ) was explored using western blotting. The effects of miR-184 inhibition on cell viability and apoptosis were explored, and the miR184 target gene was determined using a luciferase assay and western blotting. Flow cytometry and Hoechst staining were used to evaluate cell growth and apoptosis. Matrigel invasion and scratch assays were performed to measure the ability of cell invasion and migration. Results: miR-184 and HIF-1 $\alpha$ protein levels were significantly upregulated in human glioma cells. Downregulation of miR-184 inhibited cell viability and increased the HEB cell apoptotic rate. Luciferase and western blot assays verified that $\mathrm{FIH}-1$ was the target gene of miR-184 and negatively controlled the protein level of HIF-1 $\alpha$. Inhibition of HIF-1 $\alpha$ by siRNA facilitated the apoptosis of HEB cells and suppressed A172 cell invasion and migration. Conclusion: miR-184 upregulation enhanced the malignant phenotype of human glioma cancer cells by reducing $\mathrm{FIH}-1$ protein expression.
\end{abstract}

Copyright $\odot 2014$ S. Karger AG, Basel

\section{Introduction}

MicroRNA (miRNA) is a type of endogenous RNA of approximately 20-24 nucleotides. Recently, microRNAs have been widely indicated to play key roles in cell growth and apoptosis [1]. Increasing evidences suggest that approximately $70 \%$ of mammalian miRNA 
genes are located in transcription micro RNA units (TUs), most of which are located in intron regions [1]. After transcription and processing, an miRNA can bind to the complementary site of its target gene to repress gene expression by inhibiting protein translation or affecting mRNA stability [2]. In the past years, the involvement of miRNAs in cancer pathogenesis has been well established, since they can behave as oncogenes or tumor suppressor genes depending on the cellular function of their targets [3]. Recent research using high-throughput detection technology indicates that human tumor tissues have significantly different miRNA expression spectra and that the abundance of miRNAs in different cancer tissues also has obvious differences $[4,5]$.

Glioma is the most common primary brain tumor and is produced by brain and spinal cord glial cells. The annual incidence rate of glioma is approximately 3-8 people per 10 million people [6]. Similar to other tumors, glioma mainly results from genetic risk factors and environmental carcinogenic factors. Some genetic diseases, such as neurofibromatosis and tuberous sclerosis disease, are predisposing factors for glioma, and the chances of glioma are much higher in patients with these diseases than in the general population [7]. In addition, some environmental carcinogenic factors may also be associated with the occurrence of glioma. Research has shown that electromagnetic radiation, such as mobile phone use, may be related to glioma [8]. In addition, most glioblastoma patients also have cytomegalovirus infection, and most glioblastoma specimens were found to contain virally infected macrophages [9]. miRNAs have emerged as key factors involved in several biological processes, including development, differentiation, cell proliferation, and tumorigenesis [10, 11]. The dysregulation of miRNAs in cancer has been repeatedly described, for example, in prostate, bladder, and kidney cancer [12], breast cancer [13], and colon cancer [14]. Thus, it is possible that microRNA (miRNA), a highly conserved gene regulatory factor, may shed light on the potential mechanism of glioma [9].

In hypoxia, HIF contributes highly to malignant tumor survival and proliferation. HIF mainly functions as a transcriptional activator and significantly induces the expression of target genes, such as proteins inducing angiogenesis and hypoxia tolerance and inhibiting apoptosis. Thus, HIF plays key roles in tumor formation, growth, and malignant progression $[15,16]$. HIF is a heterodimer consisting of two subunits: a constitutively expressed $\beta$ subunit and an oxygen-regulated $\alpha$ subunit. To date, three $\alpha$ subunits have been identified. The HIF$1 \alpha$ and $2 \alpha$ subunits belong to the same helix-loop-helix (HLH) Per/Arnt/Sim (PAS) (bHLHPAS) family. The molecular structures of HIF- $1 \alpha$ and $2 \alpha$ subunits include bHLH-PAS (PAS mediates their combination with $\beta$ subunit to form HIF-1 and HIF-2), oxygen-dependent degradation (mediates aerobic degradation), and transcriptional activity (responsible for transcriptional activation) domains [17-19]. In comparison, HIF-3 $\alpha$ only contains the bHLH-PAS domain and is deficient in the oxygen-dependent domain. Previous studies have found that HIF- $1 \alpha$ is significantly upregulated under aerobic conditions in various cancer types [20,21]. Under hypoxic conditions, HIF-1 $\alpha$ is not degraded and is available to dimerize with constitutively present HIF- $1 \beta$ and interact with p300 in the nucleus, leading to the transcriptional activation of genes, such as those for encoding for erythropoietin and vascular endothelial growth factor [10]. Recently, HIF-1 $\alpha$ has been shown to be repressed by factor inhibiting HIF-1 (FIH-1), a protein first identified through its association with HIF$1 \alpha$ in yeast two-hybrid assays [17]. FIH-1 is well known as an asparagine hydroxylase that hydroxylates HIF, thereby inhibiting its association with p300 [19]. In other words, FIH-1 mainly functions as an inhibitor molecule necessary for HIF function. Thus, reduced FIH-1 protein levels may account for increased HIF activity in various cancers.

In this paper, we mainly focus on miR-184 because it has been proven to be dysregulated in various types of tumors $[13,14]$. Furthermore, the protein levels of HIF- $1 \alpha$ in human glioma cancer cells were found to be significantly upregulated. However, little research has studied the correlation of miR-184 and HIF- $1 \alpha$ in human glioma. Thus, this work mainly investigates miR-184 expression patterns and the correlation with HIF-1 $\alpha$ in human glioma cancer cells. 


\section{Materials and Methods}

\section{Cell culture}

Human glioma cell lines U251, TJ899, and A172 and human glia HEB cells were purchased from American Type Tissue Culture Collection. TJ861 and TJ905 were gifts from the Department of Pathology, Peking University Health Science Center and were established in our lab and maintained in DMEM supplemented with $10 \%$ fetal calf serum at $37^{\circ} \mathrm{C}$ in $5 \% \mathrm{CO}_{2}$. The U251, TJ899, A172, and HEB cells were cultured in DMEM medium containing 10\% (v/v) fetal bovine serum (FBS, HyClone), 100 IU/L penicillin, and $100 \mathrm{mg} / \mathrm{L}$ streptomycin at $37^{\circ} \mathrm{C}$ in a humidified $5 \% \mathrm{CO}_{2}$ atmosphere.

\section{Transient transfections}

miR-184 mimics, miR-184 inhibitor, or miR negative control were pre-incubated with HiperFect transfection reagent (QIAGEN) according to the manufacturer's instructions. The final concentration of microRNA analogs was $100 \mathrm{nmol} / \mathrm{L}$.

Western Blotting Analyses

Cellular proteins were extracted using RIPA buffer (SolarBio, $50 \mathrm{mM}$ Tris/HCl, pH 7.4, $150 \mathrm{mM}$ $\mathrm{NaCl} 1 \%(\mathrm{v} / \mathrm{v}) \mathrm{NP}-40,0.1 \%(\mathrm{w} / \mathrm{v}) \mathrm{SDS}$ ) containing 1\% (v/v) PMSF (SolarBio), 0.3\% (v/v) protease inhibitor (Sigma) and $0.1 \%$ (v/v) phosphorylated proteinase inhibitor (Sigma). Lysates were centrifuged at $12,000 \mathrm{rpm}$ at $4^{\circ} \mathrm{C}$ for $15 \mathrm{~min}$ and the supernatant was collected for total protein. A BCA protein assay kit (Pierce) was used to determine the protein concentration. Equal amounts of protein $(15 \mu \mathrm{g})$ was separated on an SDS-PAGE gel $(10 \%(\mathrm{v} / \mathrm{v})$ polyacrylamide) and transferred onto a PVDF membrane. Nonspecific binding was blocked using $8 \%(\mathrm{w} / \mathrm{v})$ milk in TBS-T for $2 \mathrm{hr}$ at room temperature. The membranes were then incubated with primary antibodies against GAPDH, FIH-1, HIF-1 , Bcl-2, and Bax (Cell Signaling) overnight at $4^{\circ} \mathrm{C}$. After several washes with TBS-T, the membranes were incubated in HRP-conjugated goat anti-rabbit and anti-mouse IgG or HRP-conjugated mouse antigoat IgG (Abmart, all at a 1:5000 dilution) for $2 \mathrm{hr}$ at room temperature and then washed. The target proteins were visualized using enhanced chemiluminescence (Millipore) according to the manufacturer's recommendations, and quantified using density analysis normalized against GAPDH to the manufacturer's recommendations, and expressed as the fold-change compared to control.

\section{MTT assay}

Cellviabilitywasdeterminedusinga colorimetric,3-(4,5-dimethylthiazol-2-yl)-2,5-diphenyltetrazolium bromide (MTT, Sigma) assay. Briefly, HEB cells $\left(5 \times 10^{4}\right.$ cells per well) were seeded in 96 -well tissue culture plates. At 70-80\% confluence, the cells were incubated for $16 \mathrm{~h}$ in serum-free DMEM medium. After drug treatment, the HEB cells were cultured in fresh medium containing $0.5 \mathrm{mg} / \mathrm{ml}$ MTT for a further $4 \mathrm{~h}$. The blue formazan products in the HEB cells were dissolved in DMSO and spectrophotometrically measured at a wavelength of $550 \mathrm{~nm}$.

\section{Hoechst 33258 staining}

HEB cells $\left(1 \times 10^{5}\right.$ cells per well $)$ were cultured in six-well tissue culture plates and at $70-80 \%$ confluence, the cells were incubated for $16 \mathrm{~h}$ in serum-free DMEM medium. After drug treatment, the medium was removed, and the cells were rinsed once with cold PBS and then fixed with $4 \%$ formaldehyde (Zhongshan Technology) in PBS for $15 \mathrm{~min}\left(37^{\circ} \mathrm{C}\right)$. The cells were washed three times with PBS, and the nuclei were then stained with Hoechst $33258(10 \mu \mathrm{g} / \mathrm{ml}$ ) (Sigma) for $5 \mathrm{~min}$ before being washed three times with PBS and dried.

\section{RNA isolation and real-time RT-PCR}

Total RNA was isolated with RNAiso Plus (Takara Bio) following the manufacturer's instruction. For the quantitation of miRNA, $10 \mathrm{ng}$ of the total RNA were reverse transcribed using TaqMan MicroRNA Reverse Transcription Kit (Applied Biosystems) with specific primers for miR-184 and U6, and subsequently the PCR amplifications were performed in reaction volumes of $20 \mu \mathrm{L}$ containing $10 \mu \mathrm{L}$ TaqMan $2 \times$ Universal PCR Master Mix, No AmpErase UNG (Applied Biosystems), $1 \mu \mathrm{L}$ 20× TaqMan MicroRNA Assay mix (Applied 
Biosystems) and $1.33 \mu \mathrm{L}$ template cDNA in the same system used for mRNA quantitation. The thermal cycling conditions were, a hot start step at $95^{\circ} \mathrm{C}$ for $10 \mathrm{~min}$, followed by 40 cycles at $95{ }^{\circ} \mathrm{C}$ for $15 \mathrm{~s}$ and $60{ }^{\circ} \mathrm{C}$ for 1 min. Relative miRNA expression of miR-184 was normalized against the endogenous control, U6, using the comparative delta-delta CT method.

\section{Luciferase target assay}

For luciferase assay, the 3' untranslated region (UTR) or coding region of FIH-1 including the binding sites for miR-184 was amplified from HEB cells by using the specific primers. PCR was performed with genome DNA isolated from NCTC1469 cells and the PCR product was then digested with XhoI (NEB). Then, the fragment was inserted into the XhoI-linearlized pGL3 (Promega) luciferase reporter vector. To ensure the ligation efficiency, both the PCR products and the XhoI-linearlized pGL3 vectors were treated with thermosensitive alkaline phosphatese (NEB) at $80{ }^{\circ} \mathrm{C}$ for $20 \mathrm{~min}$. Details of PCR procedures are described as follows: a hot start step at $95^{\circ} \mathrm{C}$ for $10 \mathrm{~min}$, followed by 40 cycles at $95^{\circ} \mathrm{C}$ for $15 \mathrm{~s}$ and $55^{\circ} \mathrm{C}$ for $45 \mathrm{~s}, 72$ ${ }^{\circ} \mathrm{C}$ for $30 \mathrm{~s}$. To conduct luciferase reporter assay, 5000 cells per well in a $100 \mu \mathrm{l}$ medium were seeded in 96well plates. After incubation overnight, the cells were transfected with the modified firefly luciferase vector (500 ng/ $\mu \mathrm{l}$ ) with lipofectamine TM 2000 Reagent (Invitrogen) according to the manufacturer's instruction. $48 \mathrm{~h}$ after transfection, the firefly and renilla luciferase activities were measured with the Dual-luciferase reporter assay system (Promega). To control the transfection efficiency, Firefly activity was normalized to renilla activity.

\section{Apoptosis assay}

Cells (50-60\% confluent) were transfected with miR-184 inhibitor or negative control $(40 \mu \mathrm{M}$, $30 \mathrm{~min}$ ) and washed with $1 \times$ PBS twice. Apoptosis was assessed using an Annexin-V FITC -PI Apoptosis Kit (Invitrogen, Carlsbad, CA). This assay employs fluorescein-labeled Annexin-V in concert with propidium iodide (PI) to detect the cells undergoing apoptosis. Briefly, cells were washed with $1 \times$ PBS twice and suspended at 2-3 $\times 10^{6}$ cells/mL in $1 \times$ Annexin-V Binding Buffer (10 mM HEPES/NaOH, pH 7.4, $140 \mathrm{mM}$ $\mathrm{NaCl}, 2.5 \mathrm{mM} \mathrm{CaCl}_{2}$ ). Annexin-V FITC and Propidium Iodide Buffer were added to the cells, which were then incubated at room temperature for 15 minutes in the dark. Cells were analyzed by flow cytometry (Becton Dickinson, Franklin Lakes, NJ) within 1 h of staining using the FL1 (FITC) and FL3 (PI) lines.

\section{Detection of caspase activity}

Cells transtected with HIF-1 $\alpha$-targeted siRNA or random interference siRNA in serum-free culture were collected and digested with trypsin. The cells were centrifuged at $600 \times \mathrm{g}$ at $4{ }^{\circ} \mathrm{C}$ for $10-15$ minutes and washed with PBS once. Then, cell lysate was added in the ration of 2 million cells in $100 \mu \mathrm{L}$ lysate according to the kit (Beyotime) and cell precipitation was resuspended. The supernatants were lysed on ice for 15 minutes. Then, cell lysates were centrifuged at $4{ }^{\circ} \mathrm{C}$ at $16,000-20,000 \times$ g for $10-15$ minutes and the supernatant was transferred to a centrifuge tube pre-cooled with ice bath. Next, the detection buffer was added in accordance with the reagent instruction and the sample was added and appropriately mixed. Subsequently, $10 \mu \mathrm{l} 2 \mathrm{mM}$ ice-cold Ac-DEVD-pNA was mixed at $37^{\circ} \mathrm{C}$ for $60-120$ minutes. When the color significantly changed, absorbance at $405 \mathrm{~nm}$ was measured.

\section{Matrigel invasion assay in vitro}

HEB cells were cultured in serum-free DMEM medium for 24 - $48 \mathrm{~h}$ and the cell culture was centrifuged at 12,000 rpm for $15 \mathrm{~min}$. Then, $200 \mu \mathrm{l}$ supernatant was separated as chemokine and added to the lower chamber of the Boyden chamber. The $8 \mu \mathrm{m}$ polycarbonate microporous membrane was placed between the upper and lower chambers. Suspension $(400 \mu \mathrm{l})$ of cells $\left(2 \times 10^{5}\right)$ at logarithmic growth phase was added into the upper chamber and cultured at $37^{\circ} \mathrm{C}$ with $5 \% \mathrm{CO}_{2}$ for $12 \mathrm{~h}$. Liquid in the upper chamber was removed and the non-invasion cells on the membrane surface were wiped off with wet cotton swab. After being rinsed with physiological saline and dried, the chamber was fixed with methanol for 30 min and then stained routinely with hematoxylin \& eosin. Each group had 3 parallel samples and the number of cell in 5 fields was counted under a $200 \times$ microscope. Then, the average cell number was calculated followed by statistical analysis. 
Fig. 1. Expression levels of miR-184 and HIF-1 $\alpha$ were analyzed in human glioma cells. (A) qRT-PCR analysis of miR-184 expression in human glioma cell lines U251, TJ899, A172, TJ861, and TJ905 and normal human glioma HEB cells. U6 was used as an endogenous control. (B) Analysis of HIF-1 $\alpha$ protein levels in human glioma cell lines U251, TJ899, A172, TJ861, and TJ905. The data represent the means \pm SEM of $\mathrm{n}=3$ independent experiments. ${ }^{*} P<0.05$, $* * P<0.01$ versus control.

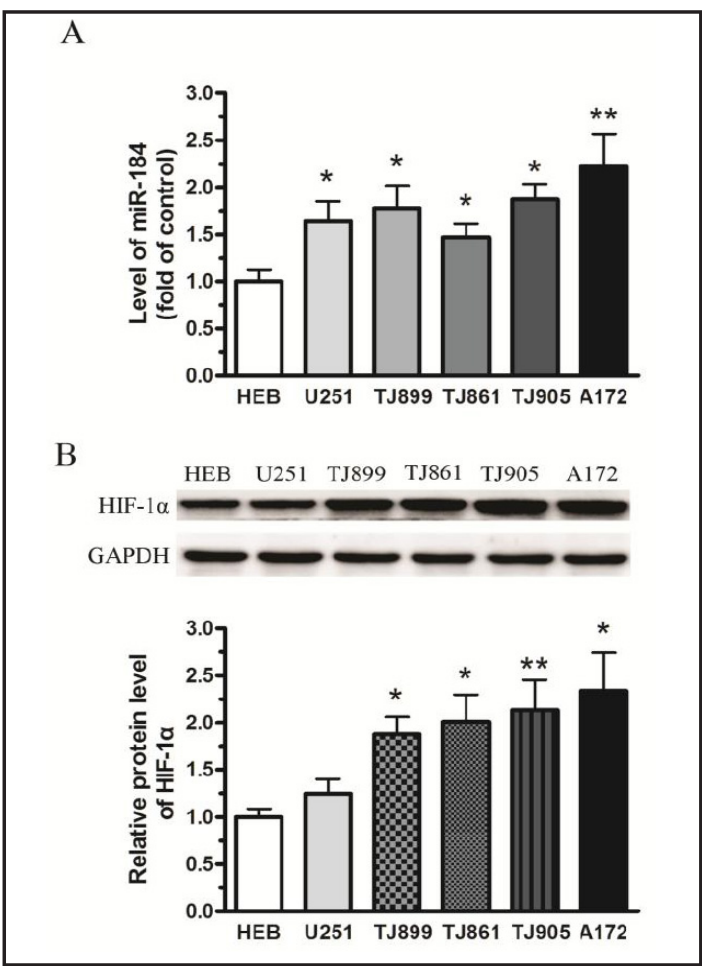

\section{Cell scratch migration test}

Cells in logarithmic growth phase were cultured in 6-well plates until confluence. Then, $200 \mu \mathrm{l}$ sterile pipetting tip was used to scratch the well bottom and PBS was used to wash off cell debris for 3 times. Then, medium containing $10 \%$ fresh fetal calf serum was added into each well, followed by incubation at $37{ }^{\circ} \mathrm{C}$ in $5 \% \mathrm{CO}_{2}$ for $24 \mathrm{~h}$ before being observed under inverted microscope. Scratch width was measured using builtin ruler of the inverted microscope $(10 \mu \mathrm{m})$. Scratch migration rate $=($ scratch width at $0 \mathrm{~h}-\mathrm{scratch}$ width at12 h) / scratch width at $0 \mathrm{~h} \times 100 \%$.

\section{Statistical Analysis}

The data were expressed as the mean \pm SEM. The number of independent experiments was represented by " $n$ ". Multiple comparisons were performed using ne-way ANOVA followed by Tukey's multiple-comparison test, where $\mathrm{P}<0.05$ was considered significant.

\section{Results}

Enhanced expression level of miR-184 and HIF-1 $\alpha$ in human glioma cells

We examined the expression level of miR-184 in human glioma cells using real-time quantitative RT-PCR. Compared with HEB cells, miR-184 was increased nearly by $64 \%, 78 \%$, $47 \%, 87 \%$, and 123\% in human glioma cell lines U251, TJ899, TJ861, TJ905, and A172, as normalized to the U6 gene (Fig. 1A). We next examined the protein levels of HIF-1 $\alpha$ in these five human glioma cancer cells. Compared with HEB cells, HIF-1 $\alpha$ was significantly upregulated in U251, TJ899, A172, TJ861, and TJ905 cells (Fig. 1B).

\section{Inhibition of miR-184 reduced HEB cell line viability}

Using a transient transfection method, human glioma cell line HEB was transfected with miR-184 mimics, inhibitor, or negative control for 24,48 , and $72 \mathrm{~h}$. In this study, the mimics were analogs that enhanced miR-184 expression level; inhibitors were analogs that decreased the expression of miR-184. As expected, when miR-184 mimics were transfected into human glioma cell line HEB, cell viability was significantly increased by $25 \%$ and $34 \%$ at $48 \mathrm{~h}$ and $72 \mathrm{~h}$, respectively (Fig. 2A). Conversely, when the miR-184 expression level was 
Fig. 2. miR-184 negatively regulated human glioma HEB cell viability. (A) HEB cells were transfected with miR-184 mimics, inhibitor (B), or negative control for 24,48 , and $72 \mathrm{~h}$. Cell viability was determined using the MTT assay. The data represent the means \pm SEM of $n=6$ independent experiments. ${ }^{*} P<0.05$ versus control.

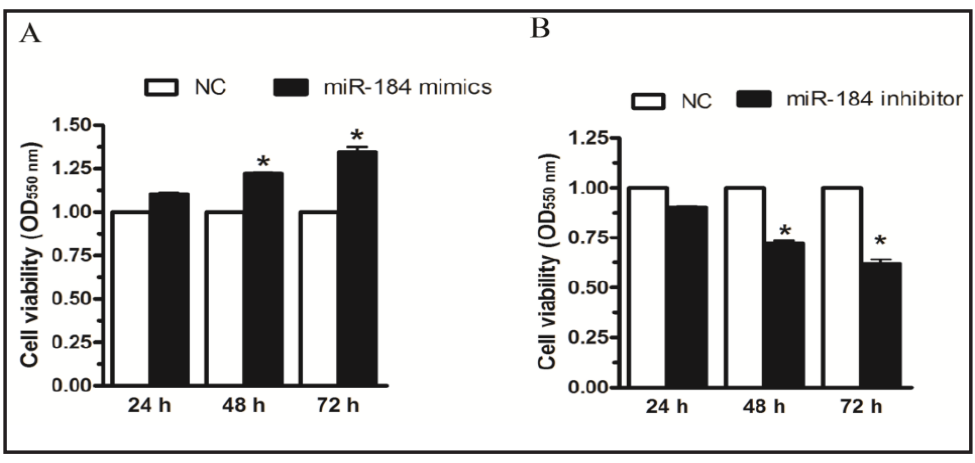

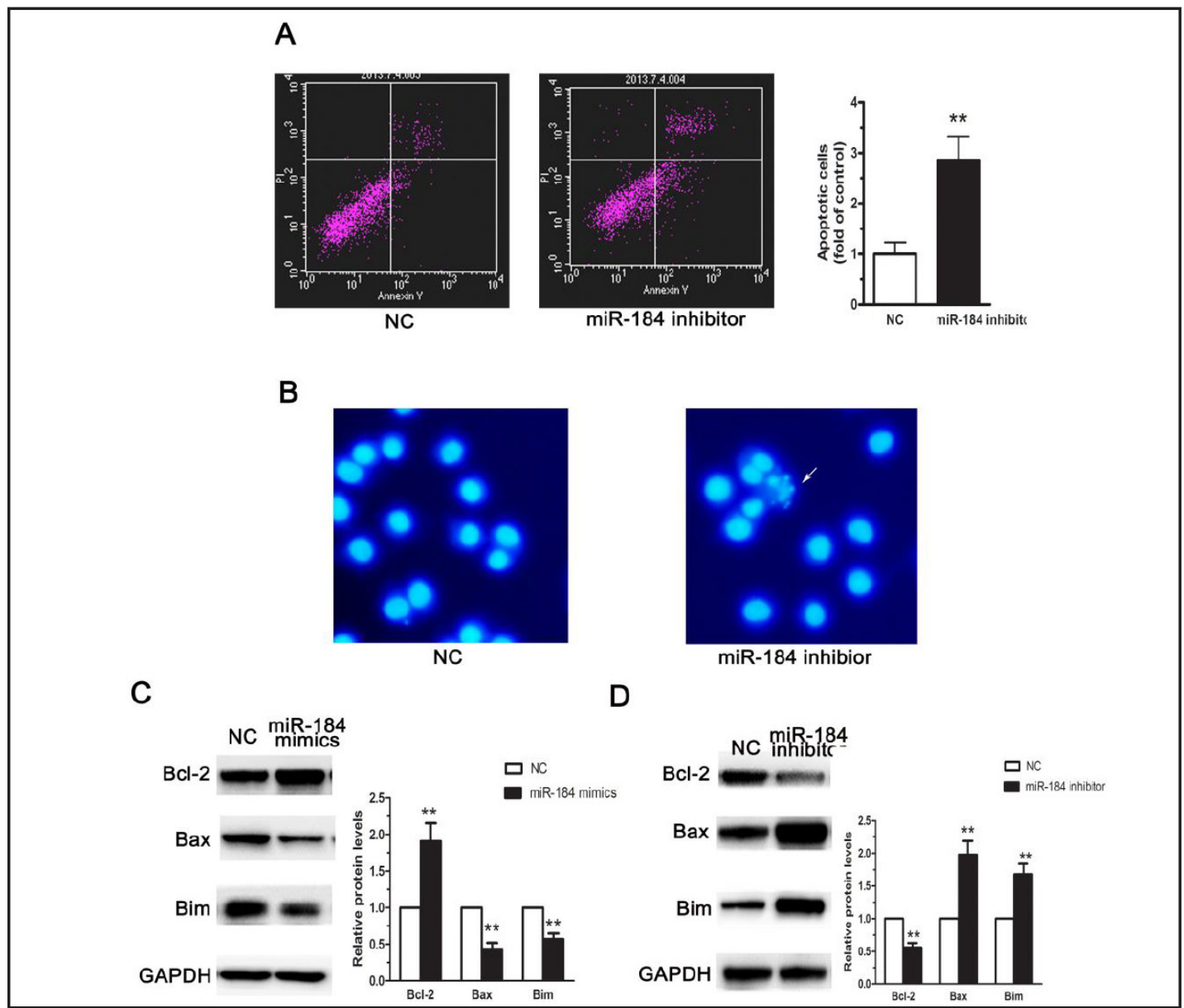

Fig. 3. Inhibition of miR-184 induced HEB cell apoptosis. Inhibition of miR-184 enhanced cell apoptosis by 1.8-fold versus the negative control in HEB cells (A), as tested using an Annexin V and PI kit. (B) Apoptotic HEB cells increased when transfected with the miR-184 inhibitor, as examined by Hoechst 33342 staining. The white arrow indicates apoptotic cells. As assessed by western blotting, the overexpression of miR-184 elevated the expression level of Bcl-2 and reduced the levels of Bax and Bim (C); the inhibition of miR-184 decreased the level of $\mathrm{Bcl} 2$ and increased the levels of Bax and Bim (D). The data represent the means \pm SEM of $\mathrm{n}=3$ independent experiments. ${ }^{* *} P<0.01$ versus control.

inhibited, cell viability was reduced by $15 \%$ and $30 \%$ at $48 \mathrm{~h}$ and $72 \mathrm{~h}$, respectively (Fig. 2B).

Inhibition of miR-184 induced HEB cell apoptosis

We next determined that the inhibition of miR-184 enhanced HEB apoptosis by nearly 1.8-fold versus the negative control (Fig. 3A). Hoechst 33342 staining also showed a higher 
A

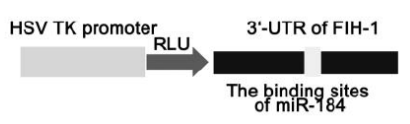

C

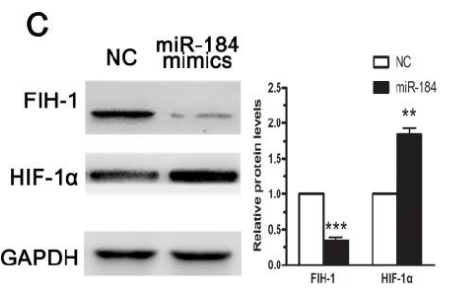

$E$

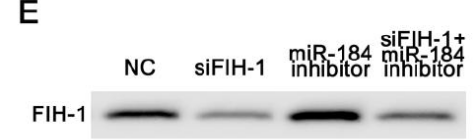

B

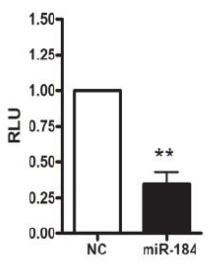

D

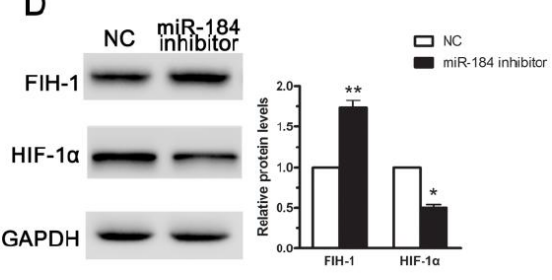

$\square$ Control

$\square$ siFHl-1

$\square$ miR-184 inhibitor

siFHI-1+miR-184 inhibitor

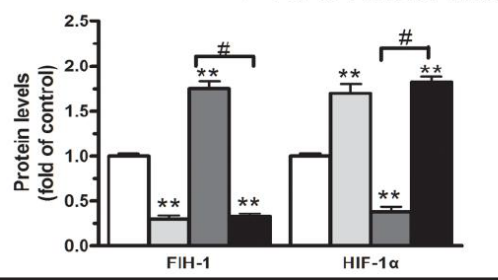

Fig. 4. miR-184 targeted FIH-1 in HEB cells. (A) The nucleotides in the 3'-untranslated region (3'-UTR) of FIH-1 from 41 bp to 47 bp in mammals are highly conserved, and this sequence was predicted to be the target site of miR-184. (B) miR-184 overexpression significantly reduced luciferase activity in HEK 293T cells transfected with the luciferase reporter vector containing the 3'-UTR region. (C) Transfection with miR-184 mimics in HEB cells decreased the expression level of FIH-1 by 70\%, and HIF- $1 \alpha$ was significantly upregulated. (D) When miR-184 was inhibited in HEB cells, the expression level of FIH-1 was increased and HIF-1 $\alpha$ was decreased. (E) Transfection with an siRNA targeting FIH-1 blocked the effects of the miR-184 inhibitor on the expression of HIF- $1 \alpha$. The data represent the means \pm SEM of $n=3$ independent experiments. ${ }^{*} P<0.05$, ${ }^{*} P<0.01$ versus control, ${ }^{*} P<0.05$ versus siFIH- $1+$ miR-184 inhibitor.

degree of apoptosis in the HEB cells transfected with an miR-184 inhibitor compared with the negative control (Fig. 3B). Furthermore, a western blot analysis of apoptotic protein expression showed that the overexpression of miR-184 restored the expression of Bcl- 2 and reduced the levels of Bax and Bim (Fig. 3C). In contrast, the inhibition of miR-184 reduced the levels of $\mathrm{Bcl} 2$ and enhanced those of Bax and Bim (Fig. 3D).

miR-184 positively regulated HIF-1 $\alpha$ expression by targeting $\mathrm{FIH}-1$

According to TargetScan, FIH-1 is predicted to be a target of miR-184. As shown in Fig. $4 \mathrm{~A}$, the nucleotides in the 3'-untranslated region (3'-UTR) of FIH-1 from $41 \mathrm{bp}$ to $47 \mathrm{bp}$ in mammals are highly conserved, and this sequence is predicted to be the target site of miR184. A luciferase reporter assay was then used to assess whether miR-184 can directly bind to the 3'-untranslated region of FIH-1. The overexpression of miR-184 significantly reduced luciferase activity in HEK 293T cells that were transfected with the luciferase reporter vector containing the 3'-UTR region (Fig. 4B). To further confirm whether miR-184 could target FIH-1, we used western blotting to detect the expression level of FIH-1. As shown in Fig. 4C, at 48 hours after transfection with miR-184 mimics, the expression level of FIH-1 in HEB cells was decreased by $70 \%$, and HIF- $1 \alpha$ was significantly upregulated (Fig. 4C). Conversely, when miR-184 was inhibited in HEB cells, the expression level of FIH-1 was increased, and HIF- $1 \alpha$ was decreased (Fig. 4D). These results indicated that FIH-1 is the target gene of miR- 
A

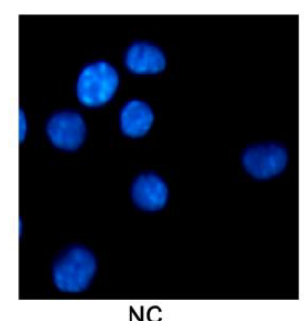

NC

B

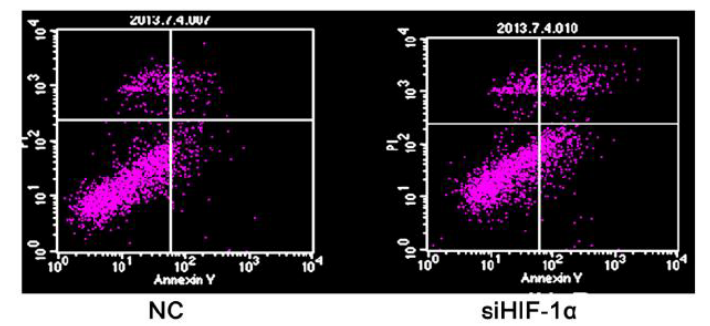

C
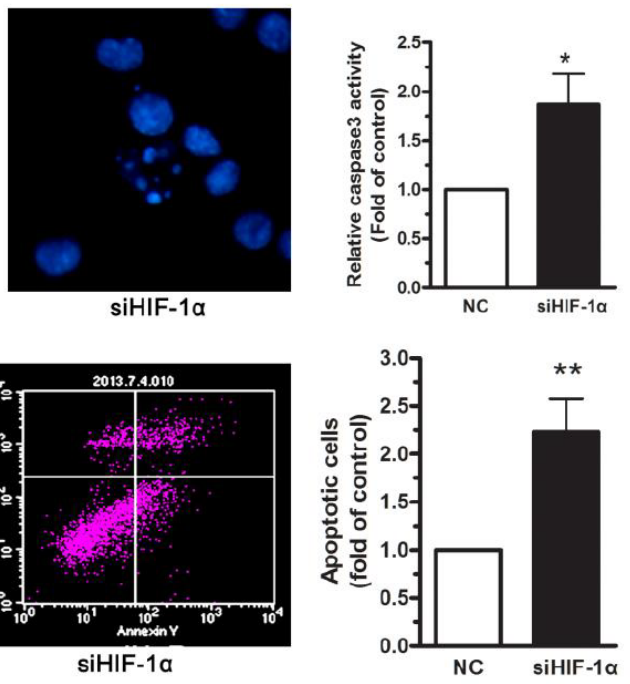

Fig. 5. Inhibition of HIF-1 $\alpha$ by siRNA enhanced apoptosis in HEB cells. (A) Hoechst 33258 fluorescence staining of cells transfected with a random siRNA or an HIF-1 $\alpha$-targeting siRNA after serum starvation for $48 \mathrm{~h}$. Cell apoptosis was detected using a Hoechst staining kit, and apoptotic cells are indicated with white arrows. (B) An HIF-1 $\alpha$-targeting siRNA enhanced cell apoptosis in HEB cells, as tested using a Annexin V and PI kit. (C) Caspase 3 activity in cell lysates of random siRNA and HIF-1 $\alpha$-targeting siRNA groups after serum starvation for $48 \mathrm{~h}$. The data represent the means \pm SEM of $\mathrm{n}=3$ independent experiments. ${ }^{*} P<0.05$, ${ }^{* *} P<0.01$ versus control.

184. We next investigated whether the downregulation of FIH-1 is an integral part of miR184-induced HIF-1 $\alpha$ overexpression. To this end, an siRNA targeting FIH-1 was selected. Transfection of the miR-184 inhibitor alone led to enhanced HIF-1 $\alpha$ expression, whereas the suppression of FIH-1 by siRNA reduced the protein level of HIF-1 $\alpha$, even in cells that were co-transfected with the miR-184 inhibitor (Fig. 4E). Taken together, these results indicate that miR-184 positively regulated HIF-1 $\alpha$ expression by targeting FIH-1.

\section{Inhibition of HIF-1 $\alpha$ by siRNA enhanced the apoptosis of HEB cells}

To explore the influence of HIF- $1 \alpha$ on HEB cell apoptosis, Hoechst staining, flow cytometry, and spectrophotometry were used. Hoechst 33258 fluorescence staining showed that the number of apoptotic cells transfected with the HIF-1 $\alpha$-targeting siRNA was significantly increased compared to that of the random siRNA group at $48 \mathrm{~h}$ of serum starvation (Fig. 5A). Consistent with this observation, flow cytometry using AnnexinV-PI staining in cells treated in the same way demonstrated that the percentage of apoptotic cells in the HIF-1 $\alpha$-targeting siRNA group was higher than that in the random siRNA group at $48 \mathrm{~h}$ (Fig. 5B). Furthermore, the detection of caspase 3 activity using spectrophotometry showed that the activity of caspase 3 in the HIF- $1 \alpha$-targeting siRNA group was higher than that in the random siRNA group (Fig. 5C). These data indicated that inhibition of HIF-1 $\alpha$ by siRNA facilitated the apoptosis of HEB cells.

\section{Inhibition of HIF-1 $\alpha$ suppressed A172 cell invasion and migration}

To test the effect of HIF-1 $\alpha$ on A172 cell invasion and migration, a Matrigel invasion assay, scratch migration test, and western blotting were performed. The in vitro invasion assay showed that the number of transmembrane cells in the HIF-1 $\alpha$-targeting siRNA group $(34.33 \pm 2.68)$ was lower than that in the random siRNA group $(82.13 \pm 5.26)$, with a statistically significant difference (Fig. 6A). The results of the scratch migration test showed that the migration rate was $15 \%$ in the cells transfected with the HIF-1 $\alpha$-targeting siRNA, 
Fig. 6. Inhibition of HIF-1 $\alpha$ suppressed A172 cell invasion and migration. (A) A172 cell invasion in random siRNA and HIF-1 $\alpha$-targeting siRNA groups. The number of transmembrane cells was counted by routine hematoxylin \& eosin staining. (B) The migration rate was determined in cells transfected with an HIF-1 $\alpha$-targeting siRNA using the scratch migration test. (C) The protein levels of MMP-2 and E-cadherin were determined using a western blot assay. The data represent the means \pm SEM of $n=3$ independent experiments. ${ }^{*} P<0.05$, ${ }^{* *} P<0.01,{ }^{* * *} P<0.001$ versus control.
A
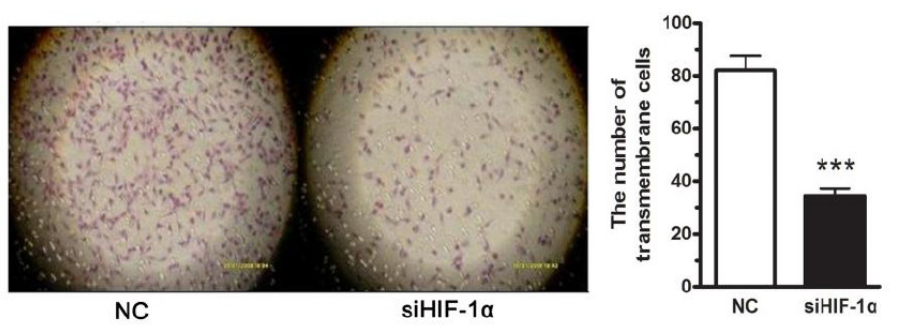

B

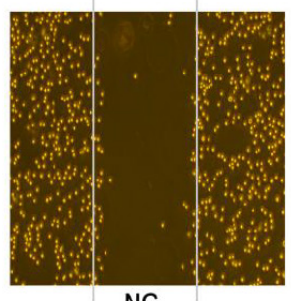

NC
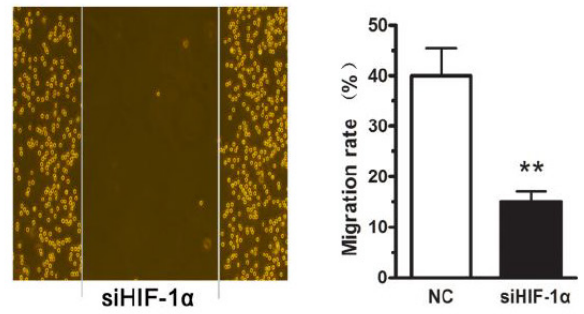

C
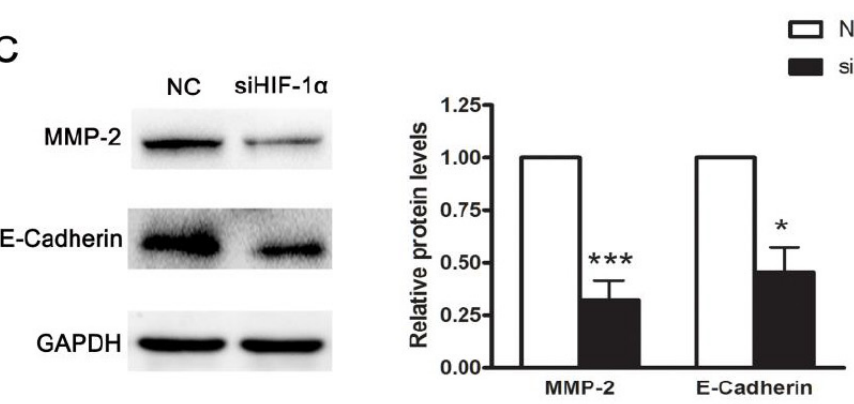

which was significantly lower than the migration rate of the random siRNA group (40\%), suggesting that reduced HIF-1 $\alpha$ protein expression significantly weakened the migration ability of the cells (Fig. 6B). Western blotting showed that MMP-2 and E-cadherin were significantly reduced in the cells transfected with the HIF-1 $\alpha$-targeting siRNA, (Fig. 6C), indicating that HIF-1 interference might induce cell migration. These data demonstrated that the inhibition of HIF-1 $\alpha$ suppressed A172 cell invasion and migration.

\section{Discussion}

miRNAs are non-coding small RNAs that mainly repress mRNA translation in cells. Through their incomplete complementary sequence with a large amount of mRNAs, miRNAs have been proven to have important functions in various oncogenic signaling pathways, such as cell proliferation and migration [22]. miRNAs can be divided into oncogenes and tumor suppressor genes according to their target genes. Glioma is the most common malignant tumor of the central nervous system, accounting for approximately $40 \% \sim 50 \%$ of all intracranial tumors [23]. However, the use of modern microsurgery, radiotherapy, chemotherapy, and other comprehensive treatment measures is still not ideal for the prognosis of glioma patients. Therefore, elucidating the molecular mechanism of glioma occurrence and development, as well as finding key signaling pathways and regulatory factors, has become the focus of brain glioma research [24].

miR-184 was reported to be widely dysregulated in human tumors [25]. In this study, we first explored the expression level of miR-184 in human glioma cells. RT-qPCR revealed that the relative quantity of miR-184 was significantly upregulated in human glioma cells, accompanied by enhanced protein levels of HIF-1 $\alpha$. Furthermore, we also demonstrated that the inhibition of miR-184 inhibited HEB cell growth and enhanced cell apoptosis. More 
importantly, we found that FIH-1 is the target gene of miR-184; by targeting FIH-1, miR-184 positively regulates HIF- $1 \alpha$ and thereby participates in tumor development and progression. During tumorigenesis and cancer progression, HIF-1 plays key roles because it helps cells to survive under low oxygen conditions [26]. HIF-1 is mainly composed of a hypoxiainducible $\alpha$ subunit and a constitutively expressed $\beta$ subunit [17]. As previous studies have indicated that HIF- $1 \alpha$ regulates angiogenesis, glucose metabolism, cellular survival, and apoptosis [15], in this study, we explored the protein levels of HIF- $1 \alpha$ in human glioma cells and found that it was significantly overexpressed. Through asparagine hydroxylation of HIF$1 \alpha$, FIH-1 blocks the binding of p300 and CBP to HIF-1, thus inhibiting HIF-mediated gene transcription $[11,12]$. Recently, FIH-1 is found to be often deleted in glioblastoma. Through repressing HIF-1 $\alpha$, FIH-1 significantly downregulates the protein levels of GLUT-1 and VEGF1 , thereby reducing the survival and angiogenesis of glioma cells under normoxic conditions [27]. A previous study has identified that miR-31 targets FIH-1 to positively regulate corneal epithelium glycogen metabolism [28]. Here, we found that FIH-1 is also a target gene of miR184.

The expression of HIF-1 $\alpha$ was significantly reduced using a specific siRNA targeting HIF$1 \alpha$, and this was accompanied by a notable growth inhibition in HEB cells. Flow cytometry and Hoechst staining confirmed that reduced HIF-1 $\alpha$ expression enhances cell apoptosis in the absence of serum starvation. HIF- $1 \alpha$ can also induce a variety of apoptosis-related proteins, such as the pro-apoptosis proteins BNIP3 and Noxa [28, 29]. IPAS is a negative regulator of HIF-1 and leads to mitochondrial depolarization and caspase 3 activation by binding to Bcl- 2 family members Bcl-xL, Bcl-w, and Mcl-1, thereby promoting cell apoptosis [30]. These data confirm the effect of HIF-1 with regard to inhibiting cell apoptosis, which mainly occurs through the mitochondria/caspase pathway.

This study further explored the invasion and migration capability of cells transfected with an HIF-1 $\alpha$-targeting siRNA. Matrigel transmembrane invasion and scratch migration tests showed that reduced HIF-1 $\alpha$ protein expression inhibited the invasion and migration ability of HEB cells. Multiple target proteins of HIF-1 are involved in invasion and migration processes, including extracellular matrix degradation target proteins such as urokinasetype plasminogen activator receptor, collagen prolyl hydroxylase, matrix metalloproteinase (e.g., MMP-2), and intercellular adhesion molecules such as E-cadherin. This study also found that the reduced expression of the HIF-1 $\alpha$ protein inhibited the expression of MMP-2 and E-cadherin, indicating the important role of HIF-1 $\alpha$ in human glioma cell invasion and migration [31].

In conclusion, our data first demonstrate the enhanced expression level of miR-184 in human glioma cells. Furthermore, we also confirmed that miR-184 underexpression inhibits HEB cell viability and induces cell apoptosis. More importantly, FIH-1 is confirmed as the direct target of miR-184 in human glioma cells. By targeting FIH-1, miR-184 positively regulates the expression of HIF-1 $\alpha$, thereby facilitating tumor survival and promoting malignant progression. The pro-survival effect of miR-184 may shed light on the treatment of human glioma cancers.

\section{Acknowledgements}

This work was supported by the National Natural Science Foundation of China.

\section{Disclosure Statement}

The authors have no conflicts of interest to declare. 


\section{References}

1 Ohgaki H, Kleihues P: Genetic alterations and signaling pathways in the evolution of gliomas. Cancer Sci 2009;100:2235-2241.

2 Stupp R, Hegi ME, Mason WP, van den Bent MJ, Taphoorn MJ, Janzer RC, Ludwin SK, Allgeier A, Fisher B, Belanger K, Hau P, Brandes AA, Gijtenbeek J, Marosi C, Vecht CJ, Mokhtari K, Wesseling P, Villa S, Eisenhauer E, Gorlia T, Weller M, Lacombe D, Cairncross JG, Mirimanoff RO; European Organisation for Research and Treatment of Cancer Brain Tumour and Radiation Oncology Groups; National Cancer Institute of Canada Clinical Trials Group: Effects of radiotherapy with concomitant and adjuvant temozolomide versus radiotherapy alone on survival in glioblastoma in a randomised phase III study: 5-year analysis of the EORTC-NCIC trial. Lancet Oncol 2009;10: 459-466.

- 3 Huse JT, Holland EC: Targeting brain cancer: advances in the molecular pathology of malignant glioma and medulloblastoma. Nat Rev Cancer 2010;10:319-331.

4 Garzon R, Calin GA, Croce CM: MicroRNAs in cancer. Annu Rev Med 2009;60:167-179.

-5 Kozomara A, Griffiths-Jones S: miRBase: integrating microRNA annotation and deep-sequencing data. Nucleic Acids Res 2011;39:D152-157.

6 Karsy M, Albert L, Tobias ME, Murali R, Jhanwar-Uniyal M: All-trans retinoic acid modulates cancer stem cells of glioblastoma multiforme in an MAPK-dependent manner. Anticancer Res 2010;30:4915-4920. Liu C, Tang DG: MicroRNA regulation of cancer stem cells. Cancer Res 2011;71:5950-5954.

8 Gulati N, Karsy M, Albert L, Murali R, Jhanwar-Uniyal M: Involvement of mTORC1 and mTORC2 in regulation of glioblastoma multiforme growth and motility. Int J Oncol 2009;35:731-740.

-9 Kefas B, Godlewski J, Comeau L, Li Y, Abounader R, Hawkinson M, Lee J, Fine H, Chiocca EA, Lawler S, Purow B: microRNA-7 inhibits the epidermal growth factor receptor and the Akt pathway and is down-regulated in glioblastoma. Cancer Res 2008;68:3566-3572.

10 Semenza GL: Targeting HIF-1 for cancer therapy. Nat Rev Cancer 2003;3:721-732.

11 Lando D, Peet DJ, Gorman JJ, Whelan DA, Whitelaw ML, Bruick RK: FIH-1 is an asparaginyl hydroxylase enzyme that regulates the transcriptional activity of hypoxia-inducible factor. Genes Dev 2002;16:14661471.

12 McNeill LA, Hewitson KS, Claridge TD, Seibel JF, Horsfall LE, Schofield CJ: Hypoxia-inducible factor asparaginyl hydroxylase (FIH-1) catalyses hydroxylation at the beta-carbon of asparagine-803. Biochem J 2002;367:571-575.

13 Kasimanickam VR, Kasimanickam RK, Dernell WS: Dysregulated microRNA clusters in response to retinoic acid and CYP26B1 inhibitor induced testicular function in dogs. PLoS One 2014;9:e99433.

14 Peng Y, Guo JJ, Liu YM, Wu XL: microRNA-34a inhibits the growth, invasion and metastasis of gastric cancer by targeting PDGFR and MET expression. Biosci Rep DOI: 10.1042/BSR20140020.

-15 Jeon HM, Sohn YW, Oh SY, Kim SH, Beck S, Kim S, Kim H: ID4 imparts chemoresistance and cancer stemness to glioma cells by derepressing miR-9*-mediated suppression of SOX2. Cancer Res 2011;71:3410-3421.

16 Jiang BH, Rue E, Wang GL, Roe R, Semenza GL: Dimerization, DNA binding and transactivation properties of hypoxia-inducible factor 1. J Biol Chem 1996;271:17771-17778.

17 Huang LE, Gu J, Schau M, Bunn HF: Regulation of hypoxia-inducible factor 1 alpha is mediated by an 0-2-dependent degradation domain via the ubiquitin- proteasome pathway. Proc Natl Acad Sci U S A 1998;95:7987-7992.

18 Makino Y, Cao R, Svensson K, Bertilsson G, Asman M, Tanaka H, Cao Y, Berkenstam A, Poellinger L: Inhibitory PAS domain protein is a negative regulator of hypoxia-inducible gene expression. Nature 2001;414:550-554.

19 Shi YH, Wang YX, Bingle L, Gong LH, Heng WJ, Li Y, Fang WG: In vitro study of HIF-1 activation and VEGF release by bFGF in the T47D breast cancer cell line under normoxic conditions: involvement of PI-3K/Akt and MEK1/ERK pathways. J Pathol 2005;205:530-536.

20 Zhang ZG, Zhang QN, Wang XH, Tian JH: Hypoxia-inducible factor 1 alpha (HIF-1 $\alpha$ ) as a prognostic indicator in patients with gastric tumors: a meta-analysis. Asian Pac J Cancer Prev 2013;14:4195-4198.

21 Zhu CL, Huang Q Liu CH, Lin XS, Xie F: Prognostic value of HIF-1 $\alpha$ expression in patients with gastric cancer. Mol Biol Rep 2013;40:6055-6062.

22 Karsy M, Arslan E, Moy F: Current progress on understanding microRNAs in glioblastoma multiforme. Genes Cancer 2012;3;3-15. 
23 Ben-Hamo R, Efroni S: Gene expression and network-based analysis reveals a novel role for hsa-mir-9 and drug control over the p38 network in glioblastoma multiforme progression. Genome Med 2011;3:77.

24 Sasayama T, Nishihara M, Kondoh T, Hosoda K, Kohmura E: MicroRNA-10b is overexpressed in malignant glioma and associated with tumor invasive factors, uPAR and RhoC. Int J Cancer 2009;125:1407-1413.

25 Sun L, Yan W, Wang Y, Sun G, Luo H, Zhang J, Wang X, You Y, Yang Z, Liu N: MicroRNA-10b induces glioma cell invasion by modulating MMP-14 and uPAR expression via HOXD10. Brain Res 2011;1389:9-18.

-26 Peng H, Hamanaka RB, Katsnelson J, Hao LL, Yang W, Chandel NS, Lavker RM: MicroRNA-31 targets FIH-1 to positively regulate corneal epithelial glycogen metabolism. FASEB J 2012;26:3140-3147.

27 Wang E, Zhang C, Polavaram N, Liu F, Wu G, Schroeder MA, Lau JS, Mukhopadhyay D, Jiang SW, O'Neill BP, Datta K, Li J: The role of factor inhibiting HIF (FIH-1) in inhibiting HIF-1 transcriptional activity in glioblastoma multiforme. PLoS One. 2014;23:e86102.

28 Bruick RK: Expression of the gene encoding the proapoptotic Nip3 protein is induced by hypoxia. Proc Natl Acad Sci USA, 2000;97:9082-9087.

29 Kim JY, Ahn HJ, Ryu JH, Suk K, Park JH: BH3-only protein Noxa is a mediator of hypoxic cell death induced by hypoxia-inducible factor 1alpha. J Exp Med 2004;199:113-124.

- 30 Torii S, Goto Y, Ishizawa T, Hoshi H, Goryo K, Yasumoto K, Fukumura H, Sogawa K: Pro-apoptotic activity of inhibitory PAS domain protein (IPAS), a negative regulator of HIF-1, through binding to pro-survival Bcl-2 family proteins. Cell Death Differ 2011;18:1711-1725.

-31 Kim MC, Cui FJ, Kim Y: Hydrogen peroxide promotes epithelial to mesenchymal transition and stemness in human malignant mesothelioma cells. Asian Pac J Cancer Prev 2013;14:3625-3630. 